Onkologe 2011 17:882-885

DOI 10.1007/s00761-011-2108-7

(c) Springer-Verlag 2011

O. Rick' P.M. Schlag ${ }^{2}$

${ }^{1}$ Klinik Reinhardshöhe, Bad Wildungen

${ }^{2}$ Comprehensive Cancer Center, Charité - Universitätsmedizin Berlin, Campus Mitte

\title{
Auf dem Weg in den Alltag
}

ende Familie, erreicht. Dadurch werden nicht nur Folgekosten für die Krankenund Sozialversicherungsträger reduziert, sondern ebenso die Selbstständigkeit, der Leistungsrahmen und die Lebenszufriedenheit der Patienten erhalten. Zusätzlich wird im Rahmen der Durchführung einer optimalen Bewegungstherapie und Umsetzung von ernährungstherapeutischen Maßnahmen sowie durch die Verbesserung des sozioökonomischen Status möglicherweise sogar eine Verbesserung des krankheitsspezifischen Überlebens erreicht.

\section{- Damit hat die onkologische \\ Rehabilitation einen wichtigen \\ Einfluss auf das Gesamtergebnis \\ der onkologischen Behandlung.}

Zur Darstellung der einzelnen Schwerpunkte im Bereich der onkologischen Rehabilitation wurden in dieser Ausgabe nicht die einzelnen Tumorentitäten gewählt, wie dies in früheren Beiträgen zur Rehabilitation in Der Onkologe der Fall war, sondern gezielt die Schwerpunkte im Hinblick auf Funktionsstörungen gesetzt. Dadurch wird deutlich, dass die Rehabilitationsmedizin ihren Blick nicht schwerpunktmäßig auf die Tumorentität, sondern auf Funktions- und Strukturstörungen sowie Einschränkungen im täglichen Leben und beruflichen Alltag legt.

Die sozialmedizinische Beurteilung als Eingangs- und Triagekriterium für eine onkologische Rehabilitation wird von $V$. König et al. vorgestellt.

Die tragende Rolle von Bewegung und Sport als Therapieform in der onkologischen Rehabilitation wird von U. Seifart et al. hervorgehoben. Hierdurch können nicht nur das Wohlbefinden wieder gestärkt und Folgestörungen bewältigt, sondern auch Prävention bezüglich des bestehenden Krebsrisikoprofils betrieben werden.

Das übergeordnet wichtige Thema „Krebs und Ernährung“ steht im Artikel von G. Faber et al. speziell auch unter den rehabilitativen Aspekten im Fokus.

Die Diagnostik und Therapie eines im Rahmen der Behandlung unterschiedlicher Tumorerkrankungen (z. B. Brustkrebs, malignes Melanom, Sarkom) möglichen Lymphödems werden im Beitrag von E.-M. Kalusche et al. besprochen.

D.-H. Zermann et al. erörtern die rehabilitativen Möglichkeiten bei funktionellen Problemen nach Therapie onkologischer Erkrankungen im Abdominal- und Beckenbereich.

O. Rick et al. widmen der Rehabilitation des lungenoperierten Tumorpatienten ein spezielles Kapitel dieses Schwerpunkthefts.

Schließlich wird auch die spezielle Rehabilitation von chemotherapieinduzierter Polyneuropathie im Beitrag von $M$. Steimann et al. besprochen.

Dem Leser wird somit ein umfänglicher Einblick in die onkologische Rehabilitationsmedizin und in die klinische Praxis einer onkologischen Rehabilitationsfachklinik gegeben. Für die praxisnahen und interessanten Beiträge der Autoren, die allesamt aus versierten onkologischen Rehabilitationskliniken kommen, möchten wir uns herzlich bedanken.

Wir sind sicher, dass ihre Mühe dazu beitragen wird, die onkologische Rehabilitation als bisher teilweise noch vernach- 
Hier steht eine Anzeige.

黛 Springer 
lässigten Pfeiler der onkologischen Therapie als unerlässliche Stütze für die Tumorpatienten auf ihrem Weg wieder zurück in den Alltag weiter mit in den Vordergrund zu rücken.

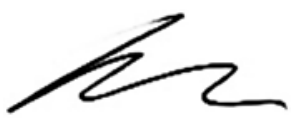

O. Rick

Für die Herausgeber des Schwerpunkthefts

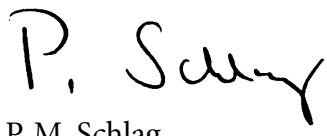

P. M. Schlag

Für die Herausgeber

\section{Korrespondenzadresse}

\section{Prof. Dr. O. Rick}

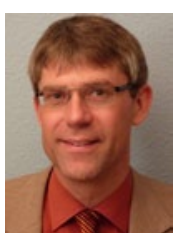

Klinik Reinhardshöhe

Quellenstraße 8-12,

34537 Bad Wildungen

oliver.rick@

klinik-reinhardshoehe.de
Der Onkologe bietet jeden Monat umfassende und aktuelle Beiträge zu interessanten Themenschwerpunkten aus allen Bereichen der Onkologie.

Möchten Sie ein bereits erschienenes Heft nachbestellen? Einzelne Ausgaben können Sie direkt bei unserem Kundenservice zum Preis von je EUR 34,- zzgl. Versandkosten beziehen:

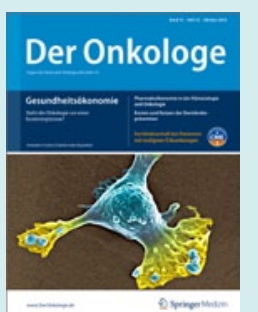

2010

- Heft $1 / 10$

- Heft 2/10

- Heft 3/10

- Heft 4/10

- Heft $5 / 10$

- Heft 6/10

- Heft $7 / 10$

- Heft 8/10

- Heft 9/10

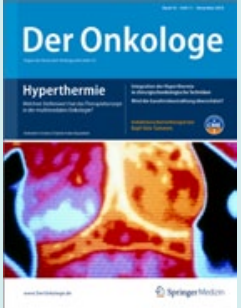

- Heft 10/10

- Heft 11/10

- Heft 12/10

Morbus Hodgkin

Nierenzellkarzinom

Myelom

Onkologische Notfälle

Ösophaguskarzinom

Pankreaskarzinom

Schilddrüsenkarzinom

Rektumkarzinom

Gallengangskarzinom

Melanom

Hyperthermie als onkologisches

Behandlungskonzept

Gesundheitsökonomie

2011

- Heft 1/11

- Heft 2/11

Hirntumoren

Krebsregister

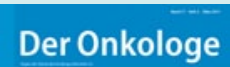

- Heft 3/11

Gesundheitsökonomie und Ethik

- Heft 4/11

Neuroonkologie

- Heft 5/11

Neue Techniken in Diagnostik und Therapie

- Heft 6/11

Molekulare Onkologie

- Heft 7/11

Neuroendokrine Tumoren

- Heft 8/11

- Heft 9/11

Nichkleinzelliges Bronchialkarzinom

Non-Hodgkin-Lymphome

- Heft 10/11

Onkologische Rehabilitation

- Heft 11/11

Tumoren im Kindesalter

- Heft 12/11

Psychoonkologie

Änderungen vorbehalten

So erreichen Sie unseren Kundenservice:

Springer Customer Service Center GmbH

Kundenservice Zeitschriften

Haberstr. 7

69126 Heidelberg

Tel.: +49 $6221345-4303$

Fax: +49 6221 345-4229

E-Mail: leserservice@springer.com

www.DerOnkologe.de 
Hier steht eine Anzeige.

黛 Springer 